\title{
Romance of the three kingdoms: RORgammat allies with HIF1alpha against FoxP3 in regulating $T$ cell metabolism and differentiation
}

\author{
Andy Tsun, Zuojia Chen, Bin Li ${ }^{\bowtie}$ \\ Key Laboratory of Molecular Virology \& Immunology, Unit of Molecular Immunology, Institut Pasteur of Shanghai, Shanghai \\ Institutes for Biological Sciences, Chinese Academy of Sciences, Shanghai 200025, China \\ $\triangle$ Correspondence: binli@sibs.ac.cn
}

Regulatory T (Treg) cells play an essential role in immune homeostasis by controlling the function of various immune effector cells, including RAR-related orphan receptor gammat ${ }^{+}$(RORyt $^{+}$) $T$ helper 17 (Th17) cells. Foekhead box $\mathrm{P}_{3}\left(\mathrm{FoxP}_{3}\right)$ is the master regulator of Treg cell function, while RORyt is the key transcription factor for the induction of the interleukin (IL)-17 family of cytokines during Th17 cell differentiation. FoxP3 can directly interact with and negatively regulate the function of RORyt, to determine the balance between induced Treg (iTreg) and Th17 cell polarization. Two recent independent studies from the Pan and Chi Labs have shown how hypoxia-inducible factor 1 alpha (HIF1 $\alpha$ ) is able to tip the balance of $\mathrm{T}$ cell differentiation toward the Th17 lineage by responding to the local changes in metabolic shift or an increase in proinflammatory mediators in the microenvironment. By allying with HIF1a, RORyt wins the fight against FoxP3 and Treg cell commitment.

The immune system is tightly controlled by the balance of multiple innate and adaptive immune cells to distinguish between self and non-self and maintain immune homeostasis. The fight between pro- and anti-inflammatory responses is often compared to the Yin-Yang philosophy, where these opposites are naturally bound but interconnect in order to maintain a healthy balance. Transcription factor networks play a central role in regulating the differentiation and function of immune cells, including T cells. In the past decade, the forkhead family transcription factor Foekhead box $\mathrm{P}_{3}\left(\mathrm{FoxP}_{3}\right)$ has drawn much attention due to its essential role in controlling the fate of the immunosuppressive regulatory $\mathrm{T}$ (Treg) cell. Another dominant player is RARrelated orphan receptor gammat (RORyt), a short isoform of the orphan nuclear receptor RORy, which is instrumental in controlling the development and function of interleukin (IL)-17 producing inflammatory effector $\mathrm{T}$ (Teff) cells known as helper T (Th) 17 cells. These transcription factors could be described as the Yin and Yang of the T cell immune system since they are intimately connected and share common differential cues; however, RORyt and FoxP3 also act reciprocally to keep one another in check.

Previously studies have shown how naïve T cells can be differentiated into induced FoxP3 $3^{+}$Treg (iTreg) cells via their co-stimulation with anti-CD3/CD28 antibodies plus transforming growth factor $\beta$ (TGF $\beta$ ) treatment, or into RORyt Th17 cells by the extra addition of the proinflammatory cytokine IL-6 (Park et al., 2005; Bettelli et al., 2006; Mangan et al., 2006; Veldhoen et al., 2006). How the balance between Th17 and iTreg cell differentiation is precisely controlled remains rather unclear, although it has been reported that FoxP3 can directly bind to RORyt to then inhibit RORyt-dependent transcriptional activation of its key target genes such as IL-17 (Zhou et al., 2008; Chen et al., 2011).

The interplay between RORyt and FOXP3 in T cell differentiation has been made clearer since the role of hypoxia and the key transcription factor, hypoxia-inducible factor 1 $\alpha$ (HIF1 $\alpha$ ), has now been identified as being essential for promoting $\mathrm{T}$ cell differentiation toward the Th17-lineage away from iTreg cell polarization (Dang et al., 2011; Shi et al., 2011). By allying with HIF1a, RORyt wins the battle against FoxP3 in favor of Th17 differentiation. HIF1a, as the third party, complicates this Yin-Yang relationship between RORyt 

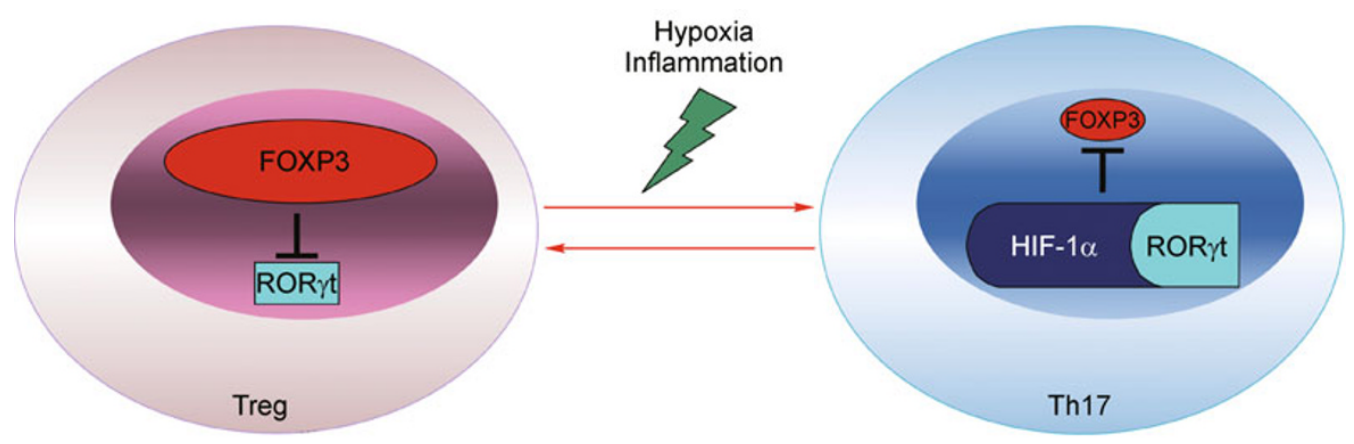

Figure 1. Regulatory $\mathrm{T}$ cells are controlled by its master transcription factor FOXP3, whereas Th17 cells utilize the combination of HIF1a and RORyt to induce its differentiation. At the protein level, FOXP3 can inhibit the function of RORyt to favor Treg differentiation, but RORyt requires the recruitment of HIF1 $\alpha$ to fight back against FOXP3 by targeting it for degradation. HIF1 $\alpha$ can also be activated by hypoxic and non-hypoxic signals to favor HIF1a/RORyt-dependent Th17 differentiation.

and FoxP3. The emergence and dynamics of the HIF1 $\alpha$ story is more akin to the interplay between the generals and armies of the Three Kingdoms era. Here, we witness the war of these transcription factors in the Th17/Treg axis (Fig. 1).

Chi and colleagues investigated the role of HIF1 $\alpha$ in regulating Tcell metabolism, focusing on glucose metabolism and its involvement in T cell differentiation. By performing in vitro $T$ cell differentiation assays, the authors found that HIF1a, which has been extensively studied in the tumor biology field as a critical regulator of cell metabolism (Finley et al., 2011), was moderately expressed in Th1 cells, highly expressed in Th17 cells, but was undetectable in Th2 or Treg cells (Shi et al., 2011). The high expression of HIF1a was found to be essential for driving the glycolytic gene expression program in Th17 cells since the HIF1 $\alpha$ deficient T cells had a much lower expression of the key transporters and enzymes including Glut1, HK2, GPI, TPI, Eno, PKM, LDHa, MCT4, and also failed to differentiate into functional IL-17 expressing Th17 cells. When naïve CD4 T cells were grown under Th17 skewing conditions, the HIF1a deficient T cells neither significantly changed the induction and expression of RORyt and RORa, nor the phosphorylation status of signal transducer and activator of transcription 3 (STAT3), but were downregulated in IL-23R expression. This resulted in the suppression of Th17 polarization, the upregulation of FoxP3 expression to further antagonize RORyt function, and the induction of the expression of Treg cell phenotypic markers such as CTLA4 and Gpr83 through TGF $\beta$ signal. By the addition of 2-Deoxy-D-glucose (2-DG), which has the 2hydroxyl group replaced by hydrogen to prevent its further glycolysis, or Rapamycin to promote the differentiation of Treg cells but not other Th subsets (Battaglia et al., 2006; Kopf et al., 2007; Haxhinasto et al., 2008; Zeiser et al., 2008; Delgoffe et al., 2011), the authors found that fewer IL-17 producing cells were generated under Th17-skewing conditions. Additionally, IL-6 promoted, but Rapamycin inhibited, the expression of HIF1a. Recently, Ramsdell and colleagues showed how FoxP3 ${ }^{+}$Treg cells and other Teff cells including Th17 cells have distinguishable differences in both glycolysis and lipid oxidation (Michalek et al., 2011). It should therefore be interesting to study whether HIF1a plays any role in suppressing lipid oxidation during Th17 and iTreg cell differentiation.

Pan and colleagues have shown how HIF1 $\alpha$ works with RORyt at multiple levels (Dang et al., 2011). Firstly, the HIF1a gene can be transcriptionally activated by the inflammatory cytokine-mediated activation of the JAK-STAT3 signal pathway that is prominent during Th17 polarization, but is independent of hypoxic stimuli. Secondly, HIF1a that is induced during $\mathrm{T}$ cell differentiation can actively promote RORyt gene transcription. Lastly, HIF1a binds to the RORyt protein complex including histone modification enzymes such as p300 which specifically functions at the promoter region of RORyt target genes (e.g. IL-17A) to induce their transcription and cell differentiation toward the Th17 cell lineage. On the other hand, HIF1 $\alpha$ interacts and regulates FOXP3 at the posttranslational level by interacting with the C-terminal domain of FOXP3 to promote its degradation in a proteasome-dependent manner. The hydroxylase targeting sites of HIF1 $\alpha$ (P402 and P564) are critical for the ability of HIF1 $\alpha$ in deregulating FOXP3, since non-proline hydroxylatable HIF1 $\alpha$ is unable to mediate FOXP3 degradation. The knockdown of the HIF1 $\alpha$ ubiquitin ligase complex subunit PHD2 can also prevent FOXP3 degradation, which suggests that HIF1 $\alpha$ may recruit its own E3 ligase complex to FOXP3. HIF1a knockout mice were found to be resistant to experimental autoimmune encephalomyelitis (EAE); here, HIF1 $\alpha$-deficient T cells were found to be resistant to hypoxia-driven Th17 cell generation in vitro, but there was an increase in the frequency of FoxP3 ${ }^{+}$ cells and degree of Treg cell differentiation in vitro and in vivo.

The three transcription factors, HIF1 $\alpha$, RORyt and FOXP3, work in conjunction with other cofactors to regulate gene transcription. HIF1 $\alpha$ acts with its heterodimer partner ARNT, which can alternatively bind to HIF1 $\beta$ (Semenza and Wang, 1992; Weidemann and Johnson, 2008), while FOXP3 can form a homodimer or heterodimerize with other subfamily 
members such as FOXP1 or multiple histone modification enzymes such as TIP60, p300 or HDAC7 in a large molecular complex (Li et al., 2006). Whether these heteromeric partners of HIF1 $\alpha$ and FoxP3 play a role in regulating their interaction with RORyt is unclear and it remains an interesting topic for future study.

The stability of HIF1 $\alpha$ itself is reversely regulated by the histone deacetylase SIRT3 (Finley et al., 2011), so the association of p300 with HIF1 $\alpha$ and RORyt may reciprocally stabilize both of these transcription factors by preventing their negative regulation by SIRT3. Moreover, FOXP3 is an acetylated protein in Treg cells (Li et al., 2007) and FOXP3 complex stability is dependent on the balance between p300 and SIRT1 activity (van Loosdregt et al., 2010); so how HIF1a can destabilize a binding partner like FOXP3 (Dang et al., 2011), but stabilize the expression of others such as RORyt (Dang et al., 2011; Shi et al., 2011) or p53 (An et al., 1998) in vivo remains an interesting issue to be solved. It is also important to understand how and whether HIF1 $\alpha$ allies with other transcription factors which are known to be essential for the differentiation and function of Th17 cells, including Batf (Schraml et al., 2009), IKB (Okamoto et al., 2010), and IRF4 (Brustle et al., 2007). IRF4 also interacts with FoxP3 and its expression is essential for FoxP3 $3^{+}$Treg cell-mediated suppression of the Th2 response (Zheng et al., 2009). It would therefore be interesting to determine whether HIF1a can regulate the function of IRF4, and how HIF1 $\alpha$ may regulate other major metabolic pathways including lipid (Michalek et al., 2011), amino acid (Cobbold et al., 2009) and nucleic acid metabolism (Tong et al., 2009) in Th17 and Treg cells.

In summary, the intriguing studies by Dang et al. and Shi et al. have highlighted the functional importance of the dynamic, temporal, and chromatin site-specific transcriptional complexes in regulating gene transcription, which leads to the precise control of crucial metabolic pathways involved in immune cell differentiation and function. The inherent differences in the metabolic pathways found in different $T$ cell types have been utilized to augment the divide between opposing $\mathrm{T}$ cell differentiation programs. HIF1a has been used by RORyt as an ally to strengthen its own game but in turn acts to ambush the FOXP3/Treg differentiation pathway. How hypoxia and inflammation work in conjunction to fine tune the balance of the Th17/Treg axis remains to be elucidated-but nonetheless, there seems to be a potential for targeting these key metabolic pathways and dynamic ensembles of transcription factor complexes for the treatment of diseases such as autoimmune disease, where Th17 and Treg cell functions are sought to be suppressed or augmented, respectively.

\section{ACKNOWLEDGEMENTS}

This study is supported by funds from NSFC (Grant Nos. 30972702 and 31050110129), SMCST09JC1416100, Shanghai Pasteur
Foundation, Shanghai "Rising Star" program 10QA1407900, China-Germany PPP program, Novo Nordisk-Chinese Academy of Sciences (NN-CAS) Foundation, CAS "100-talents" program, CAS "International Young Scientist Fellowship", and the Sanofi-AventisShanghai Institutes for Biological Sciences (SA-SIBS) scholarship program.

\section{REFERENCES}

An, W.G., Kanekal, M., Simon, M.C., Maltepe, E., Blagosklonny, M.V., and Neckers, L.M. (1998). Stabilization of wild-type p53 by hypoxia-inducible factor 1alpha. Nature 392, 405- 408.

Battaglia, M., Stabilini, A., Migliavacca, B., Horejs-Hoeck, J., Kaupper, T., and Roncarolo, M.G. (2006). Rapamycin promotes expansion of functional CD4 + CD25 + FOXP3 + regulatory T cells of both healthy subjects and type 1 diabetic patients. J Immunol 177, 8338-8347.

Bettelli, E., Carrier, Y., Gao, W., Korn, T., Strom, T.B., Oukka, M., Weiner, H.L., and Kuchroo, V.K. (2006). Reciprocal developmental pathways for the generation of pathogenic effector $\mathrm{TH} 17$ and regulatory T cells. Nature 441, 235-238.

Brüstle, A., Heink, S., Huber, M., Rosenplänter, C., Stadelmann, C., Yu, P., Arpaia, E., Mak, T.W., Kamradt, T., and Lohoff, M. (2007). The development of inflammatory $\mathrm{T}(\mathrm{H})$-17 cells requires interferonregulatory factor 4. Nat Immunol 8, 958-966.

Chen, Z., Lin, F., Gao, Y., Li, Z., Zhang, J., Xing, Y., Deng, Z., Yao, Z., Tsun, A., and Li, B. (2011). FOXP3 and RORyt: transcriptional regulation of Treg and Th17. Int Immunopharmacol 11, 536-542.

Cobbold, S.P., Adams, E., Farquhar, C.A., Nolan, K.F., Howie, D., Lui, K.O., Fairchild, P.J., Mellor, A.L., Ron, D., and Waldmann, H. (2009). Infectious tolerance via the consumption of essential amino acids and mTOR signaling. Proc Natl Acad Sci U S A 106, 12055-12060.

Dang, E.V., Barbi, J., Yang, H.Y., Jinasena, D., Yu, H., Zheng, Y., Bordman, Z., Fu, J., Kim, Y., Yen, H.R., et al. (2011). Control of T(H) $17 / \mathrm{T}(\mathrm{reg})$ balance by hypoxia-inducible factor 1 . Cell 146 , 772-784.

Delgoffe, G.M., Pollizzi, K.N., Waickman, A.T., Heikamp, E., Meyers, D.J., Horton, M.R., Xiao, B., Worley, P.F., and Powell, J.D. (2011). The kinase $\mathrm{mTOR}$ regulates the differentiation of helper $\mathrm{T}$ cells through the selective activation of signaling by mTORC 1 and mTORC2. Nat Immunol 12, 295-303.

Finley, L.W., Carracedo, A., Lee, J., Souza, A., Egia, A., Zhang, J., Teruya-Feldstein, J., Moreira, P.I., Cardoso, S.M., Clish, C.B., et al. (2011). SIRT3 opposes reprogramming of cancer cell metabolism through HIF1a destabilization. Cancer Cell 19, 416-428.

Haxhinasto, S., Mathis, D., and Benoist, C. (2008). The AKT-mTOR axis regulates de novo differentiation of CD4 + Foxp3 + cells. J Exp Med 205, 565-574.

Kopf, H., de la Rosa, G.M., Howard, O.M., and Chen, X. (2007). Rapamycin inhibits differentiation of Th17 cells and promotes generation of FoxP3+ T regulatory cells. Int Immunopharmacol 7, 1819-1824.

Li, B., Samanta, A., Song, X., Furuuchi, K., lacono, K.T., Kennedy, S., Katsumata, M., Saouaf, S.J., and Greene, M.I. (2006). FOXP3 ensembles in T-cell regulation. Immunol Rev 212, 99-113.

Li, B., Samanta, A., Song, X., lacono, K.T., Bembas, K., Tao, R., Basu, S., Riley, J.L., Hancock, W.W., Shen, Y., et al. (2007). FOXP3 
interactions with histone acetyltransferase and class II histone deacetylases are required for repression. Proc Natl Acad Sci U S A 104, 4571-4576.

Mangan, P.R., Harrington, L.E., O'Quinn, D.B., Helms, W.S., Bullard, D.C., Elson, C.O., Hatton, R.D., Wahl, S.M., Schoeb, T.R., and Weaver, C.T. (2006). Transforming growth factor-beta induces development of the $\mathrm{T}(\mathrm{H}) 17$ lineage. Nature 441, 231-234.

Michalek, R.D., Gerriets, V.A., Jacobs, S.R., Macintyre, A.N., Maclver, N.J., Mason, E.F., Sullivan, S.A., Nichols, A.G., and Rathmell, J.C. (2011). Cutting edge: distinct glycolytic and lipid oxidative metabolic programs are essential for effector and regulatory CD4+ T cell subsets. J Immunol 186, 3299-3303.

Okamoto, K., Iwai, Y., Oh-Hora, M., Yamamoto, M., Morio, T., Aoki, K., Ohya, K., Jetten, A.M., Akira, S., Muta, T., et al.(2010). IkappaBzeta regulates $\mathrm{T}(\mathrm{H}) 17$ development by cooperating with ROR nuclear receptors. Nature 464, 1381-1385.

Park, H., Li, Z., Yang, X.O., Chang, S.H., Nurieva, R., Wang, Y.H., Wang, Y., Hood, L., Zhu, Z., Tian, Q., et al. (2005). A distinct lineage of CD4 $\mathrm{T}$ cells regulates tissue inflammation by producing interleukin 17. Nat Immunol 6, 1133-1141.

Schraml, B.U., Hildner, K., Ise, W., Lee, W.L., Smith, W.A., Solomon, B., Sahota, G., Sim, J., Mukasa, R., Cemerski, S., et al. (2009). The AP-1 transcription factor Batf controls $\mathrm{T}(\mathrm{H}) 17$ differentiation. Nature 460, 405-409.

Semenza, G.L., and Wang, G.L. (1992). A nuclear factor induced by hypoxia via de novo protein synthesis binds to the human erythropoietin gene enhancer at a site required for transcriptional activation. Mol Cell Biol 12, 5447-5454.

Shi, L.Z., Wang, R., Huang, G., Vogel, P., Neale, G., Green, D.R., and Chi, H. (2011). HIF1alpha-dependent glycolytic pathway orchestrates a metabolic checkpoint for the differentiation of TH17 and Treg cells. J Exp Med 208, 1367-1376.

Tong, X., Zhao, F., and Thompson, C.B. (2009). The molecular determinants of de novo nucleotide biosynthesis in cancer cells. Curr Opin Genet Dev 19, 32-37.

van Loosdregt, J., Vercoulen, Y., Guichelaar, T., Gent, Y.Y., Beekman, J.M., van Beekum, O., Brenkman, A.B., Hijnen, D.J., Mutis, T., Kalkhoven, E., et al. (2010). Regulation of Treg functionality by acetylation-mediated Foxp3 protein stabilization. Blood 115, 965-974.

Veldhoen, M., Hocking, R.J., Atkins, C.J., Locksley, R.M., and Stockinger, B. (2006). TGFbeta in the context of an inflammatory cytokine milieu supports de novo differentiation of IL-17-producing T cells. Immunity 24, 179-189.

Weidemann, A., and Johnson, R.S. (2008). Biology of HIF-1alpha. Cell Death Differ 15, 621-627.

Zeiser, R., Leveson-Gower, D.B., Zambricki, E.A., Kambham, N., Beilhack, A., Loh, J., Hou, J.Z., and Negrin, R.S. (2008). Differential impact of mammalian target of rapamycin inhibition on CD4 + CD25 + Foxp3+ regulatory $\mathrm{T}$ cells compared with conventional CD4+ T cells. Blood 111, 453-462.

Zheng, Y., Chaudhry, A., Kas, A., deRoos, P., Kim, J.M., Chu, T.T., Corcoran, L., Treuting, P., Klein, U., and Rudensky, A.Y. (2009). Regulatory T-cell suppressor program co-opts transcription factor IRF4 to control $\mathrm{T}(\mathrm{H}) 2$ responses. Nature 458, 351-356.

Zhou, L., Lopes, J.E., Chong, M.M., Ivanov, I.I., Min, R., Victora, G.D., Shen, Y., Du, J., Rubtsov, Y.P., Rudensky, A.Y., et al. (2008). TGFbeta-induced Foxp3 inhibits $\mathrm{T}(\mathrm{H}) 17$ cell differentiation by antagonizing RORgammat function. Nature 453, 236-240. 